\title{
Acumulaciones de hierro en agroecosistemas bananeros (Milagro, Ecuador): Una revisión bibliográfica de algunos factores que intervienen en la salud y nutrición del cultivo
}

\section{Jaime Naranjo-Morán ${ }^{1,2^{*}}$, Marcos Vera-Morales ${ }^{3}$, Andy Mora-González ${ }^{4}$}

\footnotetext{
1 Escuela Superior Politécnica del Litoral, ESPOL. Centro de Investigaciones Biotecnológicas del Ecuador, CIBE. Campus Gustavo Galindo, km. 30,5 vía Perimetral, P.o. Box 09-01-5863. Guayaquil, Ecuador.

凶jaianara@espol.edu.ec

Q h https://orcid.org/0000-0002-4410-9337

${ }^{2}$ Universidad Politécnica Salesiana, Ingeniería en Biotecnología. Km 19.5 Vía a la Costa, 090901. Gua-

Q. yaquil, Ecuador.

${ }^{3}$ Escuela Superior Politécnica del Litoral, ESPOL. Centro de Investigaciones Biotecnológicas del Ecuador, CIBE. Campus Gustavo Galindo, km. 30,5 vía Perimetral, P.o. Box 09-01-5863. Guayaquil, Ecuador.

$\otimes$ mxvera@espol.edu.ec

(2) https://orcid.org/0000-0003-2342-6269

${ }^{4}$ Traviser S.A. Av. Leopoldo Carrera c s13 s/n. Guayaquil, Ecuador.

$\otimes$ andyfmoragonzalez_95@hotmail.com

Q h https://orcid.org/0000-0001-5638-6326
}

*Autor de correspondencia: jaianara@espol.edu.ec

\section{SIEMBRA}

https://revistadigital.uce.edu.ec/index.php/SIEMBRA ISSN-e: 2477-8850

ISSN: $1390-8928$

Periodicidad: semestral

vol. 8, núm. 2, 2021

siembra.fag@uce.edu.ec

DOI: https://doi.org/10.29166/siembra.v8i2.2680

Esta obra está bajo una licencia internacional Creative Commons Atribución-NoComercial

\section{Resumen}

La presente revisión bibliográfica describe desde el punto de vista agronómico las problemáticas asociadas a la acumulación de hierro $(\mathrm{Fe})$ en agroecosistemas bananeros. El objetivo de esta investigación fue analizar los factores que intervienen en la acumulación de grandes cantidades de Fe en plantaciones bananeras, a través de análisis de literatura publicados sobre este tema en distintas bases de datos, la información fue comparada con análisis foliares y suelos de una plantación bananera ubicada en la zona de Milagro, Ecuador. Los resultados muestran diversos factores que influyen en la acumulación de Fe y que el mismo puede ser transportado al follaje, para lo que el tipo de suelo es un factor determinante junto a las labores culturales que preservan el microbioma del suelo, siendo los impulsores de metabolitos sideróforos que retienen o metabolizan los metales pesados como el Fe. Los resultados de análisis foliares y de suelos muestran desequilibrios nutricionales ocasionados por factores antropogénicos y una alta bioacumulación de Fe en el cultivo de banano de la zona estudiada. En conclusión, al momento podemos comprender mejor el papel que desempeña el Fe en la plantación bananera; no obstante, se tienen que desarrollar más investigaciones de este elemento relacionado a la presión antropogénica y el tipo de suelo de la plantación. Además, es importante explorar las oportunidades biotecnológicas que pueden ofrecer los microorganismos sideróforos presentes en los agroecosistemas bananeros.

Palabras clave: banano, Fe, cultivo, nutrición, sideróforos.

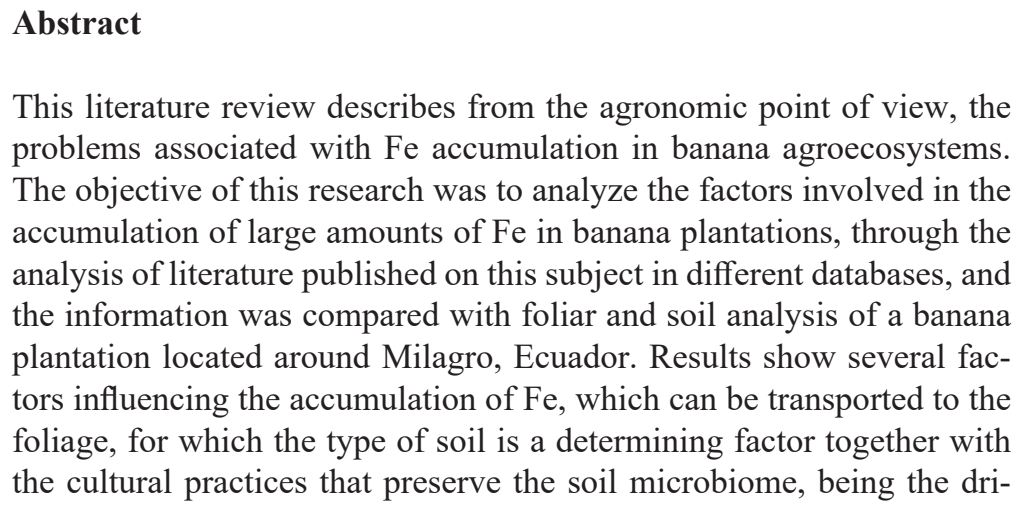


vers of siderophore metabolites that retain or metabolize heavy metals such as Fe. The results of foliar and soil analysis show nutritional imbalances caused by anthropogenic factors and a high bioaccumulation of $\mathrm{Fe}$ in the banana crop in the studied area. In conclusion, we can presently understand better the role of $\mathrm{Fe}$ in the banana plantation; however, more research on this element rela- ted to anthropogenic pressure and the type of plantation soil needs to be developed. In addition, it is important to explore the biotechnological opportunities that can be offered by siderophore microorganisms present in banana agroecosystems.

Keywords: banana, Fe, cultivation, nutrition, siderophores.

\section{Introducción}

El hierro $(\mathrm{Fe})$ es el cuarto elemento más abundante del planeta, aunque es común ubicarlo en aguas subterráneas, también puede estar presente en aguas superficiales (Baque Mite et al., 2016). La presencia en los suelos agrícolas es común ya que el hierro es un indicador del estado de oxidación de varios tipos de suelos y un reductor principal de la materia orgánica por su importante función en diferentes procesos enzimáticos, los estados de oxidación comunes son $\mathrm{Fe}^{2+}$ y Fe ${ }^{3+}$ (Grillet y Schmidt, 2017). Los niveles usuales de Fe en plantaciones bananeras son variables, no obstante, en el cinturón bananero ecuatoriano encontramos niveles de 404, 367 y 284 ppm de Fe a nivel foliar en haciendas bananeras con producción intensiva (Medina Domínguez, 2006).

En época de sequía, cuando se extrae agua de acuíferos, el hierro entra en contacto con el aire y se oxida (Martínez et al., 2003), lo que podría desencadenar desequilibrios nutricionales al precipitarse en los suelos cultivados de banano. El Fe está disponible para la planta de banano conforme al pH del suelo (Sancho y Molina, 2016). En suelos ácidos, el Fe se encuentra fácilmente disponible, mientras que en suelos alcalinos o neutros es insoluble y se observan deficiencias en las plantas (Hedrich et al., 2011). Por lo general, se piensa que el Fe no genera mayores desafíos en las plantaciones bananeras y se desestima su manejo e importancia en el agroecosistema (Kumar et al., 2011).

$\mathrm{El} \mathrm{Fe} \mathrm{en} \mathrm{el} \mathrm{suelo} \mathrm{puede} \mathrm{estar} \mathrm{retenido} \mathrm{por} \mathrm{el} \mathrm{fósforo,} \mathrm{formando} \mathrm{fosfatos} \mathrm{de} \mathrm{hierro,} \mathrm{lo} \mathrm{cual} \mathrm{repercute} \mathrm{tanto} \mathrm{en}$ la dinámica sedimentaria de estos depósitos como en las comunidades microbianas (Sulu-Gambari, 2016). Los técnicos de campo y productores bananeros le restan importancia a la revisión de los niveles acumulados de Fe en el cultivo, dado que es un micronutriente esencial para las plantas. No obstante, el conocer los niveles adecuados de este elemento en el cultivo de banano permitiría orientar adecuadamente a los profesionales agrícolas para lograr una mayor productividad. Una producción óptima de 50 toneladas de fruta de banano requiere de $900 \mathrm{~g} / \mathrm{ha} /$ año de Fe en el suelo (Lahav y Turner, 1992). Los desequilibrios nutricionales y microbianos no solo son generados por la génesis del suelo sino por malas prácticas agrícolas, como las aplicaciones excesivas de fertilizantes, sumado a la falta de tecnificación en los cultivos (Espinosa y Mite, 2008), lo que afecta de forma directa en la salud del agroecosistema, con una baja productividad y menores ingresos a los productores bananeros.

El Fe es un elemento útil para las plantas porque cataliza la estructuración de la clorofila y funciona como un trasportador de oxígeno. La homeostasis del $\mathrm{Fe}$ en las plantas contribuye a los cambios en la permeabilidad de la endodermis de la hoja (Connorton et al., 2017). En los frutos de banano la biodisponibilidad del Fe está centrada en la ferritina, siendo de gran importancia para la valoración nutricional, dado que el Fe puede estar presente en distinta concentración en esta fruta, según su procedencia o estilo de cultivo (orgánico o convencional) (Forster et al., 2002; Zielinska-Dawidziak, 2015).

El Fe en el agroecosistema desempeña un papel funcional en la disminución de la afectación de enfermedades como la fusariosis ( $F$. oxysporum) y las respuestas inmunitarias a éste y otros patógenos rizosféricos (Dong et al., 2016). El Fe puede estimular la producción de sideróforos en el suelo, por parte de bacterias como B. subtillis, P. chrysogenum, P. fluorescens y H. capsulatum (De Serrano et al., 2017; Kesaulya et al., 2018). Los sideróforos microbianos son moléculas secretadas cuando existe deficiencia de Fe en el entorno. En algunas bacterias el Fe es parte esencial de su crecimiento y respuesta al estrés oxidativo (Pi y Helmann, 2017). En este sentido, las comunidades microbianas tienen un rol fundamental para desarrollar una oxidación efectiva, haciendo que el Fe se encuentre disponible para las plantas, al oxidarse perdiendo un electrón $\left(\mathrm{Fe}^{3+}\right.$ a $\left.\mathrm{Fe}^{2+}\right)($ Hedrich et al., 2011). Además de las bacterias, otros organismos del suelo, como las micorrizas arbusculares y lombrices de tierra, pueden ser utilizados para restaurar ambientes perturbados (Khatua et al., 2018; Gbongue et al., 2019). Por ejemplo, el uso de los inoculantes microbianos representan una alternativa para mitigar los impactos ambientales (Santos et al., 2019) y mejorar las tasas de supervivencia de plántulas de banano micropropagadas (Madaan et al., 2013). Dado 
que una nutrición adecuada promoverá una protección efectiva contra las enfermedades y un mejor equilibrio de las comunidades microbianas nativas (Phirke et al., 2008; Usuga Osorio et al., 2008).

La acumulación de Fe en plantaciones de banano es influenciada por las condiciones ambientales y antropogénicas, lo que permite evidenciar las limitaciones, oportunidades de manejo e investigación sobre este micronutriente en el sector bananero de Ecuador. La toxicidad por exceso de Fe en el cultivo de banano es poco común, en ella se puede observar síntomas en hojas viejas como necrosis marginal, en suelos arcillosos con escaso oxígeno presente y de temporalidad lluviosa (Lora Silvia, 2007). El ciclo de reducción-oxidación del $\mathrm{Fe}$ es un factor vital para el desarrollo del agroecosistema bananero, ya que el $\mathrm{Fe}$ es multifuncional para todos los organismos vivos. En presencia de agua y oxígeno está presente como $\mathrm{Fe}^{3+}$, en particular como formas hidratadas no disponibles para las plantas en suelos con características alcalinas o calcáreas. El mecanismo central de la absorción de Fe está en la raíz mediante diferentes estrategias basadas en quelación y la secreción de fitosideróforos, la primera relacionada a plantas de la familia Poaceae y la segunda a la familia Fabaceae (Grillet y Schmidt, 2017), lo que en el caso de las musáceas, como el banano, es posible que derive de su expresión genética del cultivo para la adquisición de Fe según las condiciones edáficas predominantes (Tsai y Schmidt, 2020). El objetivo de esta revisión fue analizar los factores que intervienen en la acumulación de Fe en plantaciones bananeras, dado que es un micronutriente esencial para las plantas, pero que en exceso puede producir efectos tóxicos.

\section{Materiales y métodos}

En la presente revisión se utilizaron cinco bases de datos: Google Scholar, ScienceDirect, SciELO, PubMed y Springer para obtener artículos de importancia sobre la presencia de Fe en agroecosistemas bananeros, haciendo uso de términos claves como "hierro", "banano", "suelo" y "sideróforos". Las etapas del fichaje crítico incluyeron leer resúmenes, títulos relacionados, textos completos de artículos, interpretación de resultados de suelos y foliares, extracción y análisis de datos especializados. La recopilación fue limitada para artículos de investigación publicados en idioma inglés y español en el periodo 2000-2020, con el fin de evidenciar las principales problemáticas asociadas al manejo del cultivo de banano en las últimas dos décadas.

Para contrastar la información recopilada se obtuvieron datos e información de análisis foliares y de suelo de tres sectores de la plantación bananera San Valentín, ubicada en la parroquia Mariscal Sucre, Milagro, perteneciente a la provincia del Guayas, Ecuador $\left(2^{\circ} 07^{\prime} 26^{\prime \prime S}\right.$ y $79^{\circ} 29$ 22»» W). El muestreo de suelo se realizó con una pala, a una profundidad de $0-20 \mathrm{~cm}$ de la superficie del suelo, siguiendo un muestreo en zigzag, en tres lotes de la plantación, en cada uno de los cuales se tomó una muestra compuesta de 10 submuestras. Las submuestras se tomaron a 10 plantas prontas a la floración con un hijo de sucesión de 1,5 m (Instituto Nacional de Investigaciones Agropecuarias [INIAP], 2013). Para el muestreo foliar se seleccionó la tercera hoja de arriba hacia abajo, en la cual se cortaron $30 \mathrm{~cm}$ de limbo, en la sección media de la hoja a cada lado de la nervadura central. El muestreo foliar tuvo el mismo número de submuestras que el muestreo de suelos. Las plantas seleccionadas estuvieron en estado de desarrollo previo a la floración.

Los análisis de suelos y foliares fueron realizados en el laboratorio de suelos y aguas del Instituto Nacional de Investigaciones Agropecuarias (INIAP), estación experimental Litoral Sur en el mes de enero del año 2020, con el fin de cuantificar los parámetros de pH, materia orgánica (MO), capacidad de intercambio catiónico (CIC), macronutrientes ( $\mathrm{N}, \mathrm{P}, \mathrm{K}, \mathrm{Ca}, \mathrm{Mg}, \mathrm{S}$ ) y micronutrientes ( $\mathrm{Zn}, \mathrm{Cu}, \mathrm{Fe}, \mathrm{Mn}, \mathrm{B}$ ) para el suelo y los dos últimos parámetros para análisis foliar. Las muestras fueron analizadas mediante metodologías colorimétricas y técnicas de absorción atómica de elementos químicos. Estas metodologías proporcionan resultados analíticos para determinar el estado nutricional del agroecosistema bananero. La interpretación de estos resultados analíticos es fundamental, dado que conllevan a la optimización de los planes de fertilización sectorizados y así evitar la degradación química del suelo ocasionado por las malas prácticas agrícolas que soporta este agroecosistema (Pérez-López, 2013). Vale la pena decir que el análisis del follaje en el cultivo de banano es de vital importancia para comprender los procesos relacionados con la absorción de nutrientes, así como la bioacumulación de los elementos presentes en la superficie del terreno y una mejora en la toma de decisiones en el manejo cultural y en las aplicaciones de fertilizantes y agrotóxicos (Agrocalidad, 2015).

Esta información es clave para determinar un diagnóstico oportuno, ya que la tasa de absorción y distribución varía según las sustancias nutritivas aplicadas o encontradas en el suelo. El muestreo continuo permite planificar, orientar y sustituir fertilizantes para lograr una nutrición adecuada (de Deus et al., 2018). Motivo 
por el cual se cuantificó el factor de bioacumulación de elementos para determinar la capacidad de absorción de la planta sobre la cantidad de nutrientes presentes en el suelo cultivable (El-Amier et al., 2017). El factor de bioacumulación fue calculado a partir de la cantidad del elemento absorbido por la planta, dividido entre la cantidad del elemento presente en el suelo (Maldonado-Magaña et al., 2011).

\section{Resultados y discusión}

\subsection{Manejo agronómico del hierro en una plantación bananera}

La planta de banano responde a la aplicación nutricional sinérgica de tipo Liebig, que estimulan a las deficiencias (Rietra et al., 2017), no obstante, las combinaciones de fertilizantes deben ser adecuadas o no a las condiciones edafoclimáticas (Aulakh y Malhi, 2005). El uso eficiente de fertilizantes inicia desde la realización de análisis de suelo y un diagnóstico de campo pertinente a la plantación establecida, puesto que de éste depende la cantidad de los nutrientes en el suelo y su disposición al sistema radicular del cultivo de banano.

Siempre se han recomendado aplicaciones lo más cercanas a las zonas de mayor asimilación, para así lograr una mayor homeostasis del Fe en el crecimiento de las plantas (Abadía et al., 2011). Sin embargo, este panorama no siempre se da de forma satisfactoria en las plantaciones bananeras, sobre todo en aplicaciones desmesuradas por desconocimiento técnico del fertilizante, esto puede tener efectos contraproducentes en suelos neutros o ligeramente ácidos (Karimian et al., 2018). En efecto, el origen de los nutrientes con los que fertilizamos puede tener altos niveles de trazas de $\mathrm{Fe}$, en particular los fertilizantes edáficos de fuentes minerales, éstos son suministrados continuamente al suelo y pueden causar desequilibrios nutricionales severos a los suelos de tipo arcilloso (Wang et al., 2018; Van Groeningen et al., 2020).

La materia orgánica puede contribuir a la disminución del $\mathrm{pH}$ del suelo y aumentar el contenido de $\mathrm{Fe}$ en formas más complejas (Tan et al., 2019). El pH influye en la absorción de agua y nutrientes, por lo que aplicaciones altas de un elemento tienen un efecto directo en el sinergismo o antagonismo del $\mathrm{Ca}, \mathrm{K}$ y $\mathrm{Mg}$ (Sancho y Molina, 2016). La aplicación de fertilizantes orgánicos a largo plazo contribuye a la movilización de los minerales de Fe al suelo cultivable, a causa de la interacción entre los materiales orgánicos y los minerales de Fe reactivos, que permiten una mayor conservación del carbono presente en el coloide del suelo (Huang et al., 2016).

El agua de riego está cargada de metales como el Fe. Vale la pena decir que una eficiente programación del riego puede contribuir a un manejo oportuno de la plantación (Caicedo Camposano et al., 2015). El Fe y otros metales pueden ingresar por el agua de riego, así como también por las aplicaciones continuas o excesivas de fertilizantes y agrotóxicos (Sahodaran y Ray, 2018). La planta de banano en su fase de desarrollo absorbe alrededor de 1-3 g de Fe. A pesar de ser abundante el Fe en el suelo, las deficiencias de este elemento se observan en suelos de tipo calcáreo, con alto contenido de manganeso y suelos mal drenados (Bustamante León et al., 2018). En ocasiones el Fe no es fácilmente asimilable por las plantas, dado que tiene una rápida oxidación de $\mathrm{Fe}^{2+}$ a $\mathrm{Fe}^{3+}$ formando compuestos insolubles. Esto permite que en los suelos puedan formarse óxidos e hidróxidos de hierro con diferentes composiciones y grados de cristalización y, por tanto, distinta solubilidad (Aguado-Santacruz et al., 2012).

Las raíces de banano se encuentran a una profundidad de $70 \mathrm{~cm}$, la mayor parte corresponden al anclaje y el resto de las raíces más someras, permitiendo una constante absorción de nutrientes disponibles (Rodríguez et al., 2005). La compactación del suelo no permite la precipitación del Fe a las capas más profundas, evita la penetración de las raíces y limita la oxigenación de las comunidades microbianas (Olivares et al., 2018). Al oxigenarse mediante la remoción física del suelo o la elaboración de canales a una mayor profundidad, se proporciona mayor liberación de fosfatos biodisponibles para las plantas, dado que el Fe se precipita a las capas freáticas, lo que genera una mayor eficiencia en la absorción de los productos fosfatados aplicados a la plantación. Un mal manejo del suelo asociado a un plan de fertilización que no considera la innovación ni avances tecnológicos estandarizados en los países bananeros afecta drásticamente la toma de decisiones oportunas en las plantaciones bananeras, a consecuencia de esto la planta toma el nutriente que necesita y el resto se bloquea o bien se lixivia, generando pérdida en el rendimiento y productividad, por tal razón se debe trabajar con criterio técnico y científico para lograr avances significativos en la producción de banano (Izquierdo y Armas, 2018). 


\subsection{Análisis de nutrientes en una plantación bananera}

La plantación bananera San Valentín tiene un suelo ligeramente ácido (Tabla 1), lo que permite interpretar que el Fe podría estar fácilmente disponible para las plantas (Hedrich et al, 2011). Además, se encontró que existe un desequilibrio de bases perjudicial para el cultivo de banano, a consecuencia de la alta concentración de micronutrientes, como es el caso del Zn en comparación al P disponible (Raghupathi et al., 2002; Rietra et al., 2002).

Tabla 1. Análisis químico del suelo de la hacienda bananera San Valentín.

Table 1. Chemical analysis of soil from San Valentín banana farm.

\begin{tabular}{cccc}
\hline Parámetros & Sector $\mathbf{1}$ & Sector $\mathbf{2}$ & Sector 3 \\
\hline $\mathbf{p H}$ & 6,80 & 6,76 & 6,57 \\
$\mathbf{M O}(\mathbf{\%})$ & 3,14 & 2,80 & 2,91 \\
$\sum$ Bases - CIC ${ }^{*}$ & 17,31 & 14,74 & 15,65 \\
$\mathbf{C a} / \mathbf{M g}$ & 7,13 & 3,27 & 4,95 \\
$\mathbf{M g} / \mathbf{K}$ & 10,19 & 16,00 & 12,03 \\
$\mathbf{C a} / \mathbf{K}$ & 72,63 & 52,36 & 68,23 \\
\hline
\end{tabular}

*CIC = Capacidad de intercambio catiónico.

Los análisis foliares y de suelos son la base para un manejo agronómico adecuado del agroecosistema bananero, éstos en repetidas ocasiones son realizados por los productores bananeros resguardando la trazabilidad de sus lotes. Los mismos que pueden evidenciar altas concentraciones de Fe, Mn, B y Zn (Tabla 2). Se sabe que una pequeña parte del Fe presente en el suelo es acumulada en la biomasa foliar, estos resultados coinciden aun cuando se utilizan sustratos enriquecidos con Fe-EDTA (Oliveira et al., 2014), dado que las plantas pueden producir nanocomplejos de Fe en la superficie de la raíz para fitoestabilizar y contrarrestar el exceso de este elemento en el suelo (Pardha-Saradhi et al., 2014). El factor de bioacumulación del Fe en diferentes plantas es inferior a 0,4 (El-Amier et al., 2017). Sin embargo, los resultados observados en la plantación bananera San Valentín supera 3,6 de bioacumulación de Fe, notándose además una alta acumulación de B, Mn, Zn y K, éste último debido a la gran demanda en el cultivo. Los resultados del análisis de suelos muestran la falta de este elemento en el coloide del suelo, por lo que requiere aplicación inmediata en esta plantación. Los resultados fueron contrastados con los niveles óptimos del análisis foliar y análisis de suelo de una plantación bananera de alto rendimiento (López y Espinoza, 1995).

Tabla 2. Análisis de nutrientes en suelo y a nivel foliar (hoja 3) y factores de bioacumulación en tres sectores de la hacienda bananera San Valentín.

Table 2. Soil and leaf 3 nutrient and bioaccumulation factors analysis in three sectors of San Valentin banana farm.

\begin{tabular}{|c|c|c|c|c|c|c|c|c|c|c|c|}
\hline \multirow[b]{2}{*}{$\begin{array}{l}\text { Elementos } \\
(\mathrm{mg} / \mathrm{kg})\end{array}$} & \multicolumn{3}{|c|}{ Sector 1} & \multicolumn{3}{|c|}{ Sector 2} & \multicolumn{3}{|c|}{ Sector 3} & \multicolumn{2}{|c|}{ Valores Óptimos } \\
\hline & $\mathbf{A F}$ & AS & FBC-1 & $\mathbf{A F}$ & AS & FBC-2 & $\mathbf{A F}$ & AS & FBC-3 & $\mathbf{A F}$ & AS \\
\hline $\mathbf{N}$ & 2,72 & 0,54 & 5,037 & 2,56 & 0,58 & 4,414 & 2,39 & 0,57 & 4,193 & 2,6 & $0,2-0,25$ \\
\hline $\mathbf{P}$ & 0,18 & 11,77 & 0,015 & 0,20 & 7,14 & 0,028 & 0,19 & 8,95 & 0,021 & 0,2 & $10-40$ \\
\hline $\mathbf{K}^{*}$ & 2,87 & 0,20 & 14,350 & 3,02 & 0,20 & 15,100 & 2,95 & 0,21 & 14,048 & 3,0 & $0,2-1,5$ \\
\hline $\mathrm{Ca}$ & 0,60 & 14,53 & 0,041 & 0,72 & 10,68 & 0,067 & 0,75 & 12,54 & 0,060 & 0,5 & $4-20$ \\
\hline Mg & 0,44 & 2,04 & 0,216 & 0,50 & 3,26 & 0,153 & 0,48 & 2,35 & 0,204 & 0,3 & $1-10$ \\
\hline $\mathbf{S}$ & 0,21 & 168,09 & 0,001 & 0,15 & 62,43 & 0,002 & 0,19 & 89,15 & 0,002 & 0,23 & $5-50$ \\
\hline $\mathrm{Zn} *$ & 16,83 & 8,18 & 2,057 & 15,23 & 9,42 & 1,617 & 15,52 & 9,01 & 1,723 & 18 & $3-15$ \\
\hline $\mathrm{Cu}$ & 10,76 & 6,67 & 1,613 & 11,12 & 5,23 & 2,126 & 11,78 & 6,87 & 1,715 & 9 & $1-20$ \\
\hline $\mathrm{Fe}^{*}$ & 313,35 & 85,10 & 3,682 & 273,75 & 70,79 & 3,867 & 298,68 & 81,52 & 3.664 & 80 & $10-50$ \\
\hline Mn* & 118,92 & 37,66 & 3,158 & 85,67 & 28,66 & 2,989 & 94,63 & 32,51 & 2.911 & 25 & $5-50$ \\
\hline B* & 19,59 & 0,93 & 21,065 & 22,29 & 1,00 & 22,290 & 20,84 & 0,98 & 21.265 & 11 & $6-60$ \\
\hline
\end{tabular}

$\mathrm{AF}=$ Análisis foliar, $\mathrm{AS}=$ Análisis de suelo, $\mathrm{FBC}=$ Factor de bioacumulación * Elementos con $\mathrm{FBC}>2$. 
Las aplicaciones excesivas de micronutrientes en la plantación bananera producen desequilibrio de nutrientes como es el caso del P y Zn (Raghupathi et al., 2002). Lo que sugiere un desequilibrio inducido por aplicaciones no adecuadas de estos elementos en la plantación San Valentín, sumado a la gran cantidad de Fe que se encuentra en el suelo, lo que puede generar una toxicidad que se caracteriza por necrosis marginal en las hojas viejas. La mejor opción para mitigar los desequilibrios del presente agroecosistema es revertir esta situación, al definir una estrategia nutricional efectiva al cultivo de banano, como plan o programa de fertilización que integre el uso de enmiendas orgánicas (Izquierdo y Armas, 2018), en adición a los cambios sugeridos de las labores culturales para evitar la compactación del suelo cultivado.

\subsection{Sideróforos microbianos para la captación de Fe}

Los microorganismos y las plantas pueden generar sustancias metabólicas (sideróforos y fitosideróforos) para la captación de Fe y son un factor de gran importancia para el estudio de las interacciones de los nutrientes y contaminantes del suelo (Kramer et al., 2019; Honda y Borthakur, 2020). Estos metabolitos liberan el Fe de las reservas ambientales y lo envían a las células a través de receptores particulares. Los sideróforos son mediadores de las interacciones entre conjuntos microbianos y los hospederos eucariotas donde habitan (Kramer et al., 2019). Algunos han sido aplicados en la industria de alimentos, medicina, agricultura y biorremediación (De Serrano, 2017). Los sideróforos también son producidos en ambientes extremos (De Serrano et al., 2016). Aunque la mayoría no se han caracterizado en su estructura y fisiología (Årstøl y Hohmann-Marriott, 2019).

En las plantas de banano la enfermedad moko es regulada de forma distinta por la síntesis de sideróforos (Ailloud et al., 2016), por tanto, el Fe puede desempeñar un papel importante proporcionando resistencia contra los patógenos (Herlihy et al., 2020). Un caso particular, es el hongo Alternaria alternata, que utiliza dos sistemas sideróforos para la adquisición de $\mathrm{Fe}$, debido a que la disponibilidad y el metabolismo del Fe están íntimamente relacionados con su desarrollo y virulencia (Voß, 2020). En el caso de bacterias, el sideróforo conocido como pioverdina es sintetizado por Pseudomonas, contribuyendo a un mayor crecimiento y rendimiento de cultivos de importancia comercial (Sulochana et al., 2014) (Tabla 3).

Tabla 3. Sideróforos y fitosideróforos publicados en revistas científicas de alto impacto.

Table 3. Siderophores and phytosiderophores published in high-impact scientific journals.

\begin{tabular}{|c|c|c|c|}
\hline Sideróforos & Microorganismos & Cultivos asociados & Referencias \\
\hline Bacilibactina & Bacillus subtilis & Cucumis sativus & Hördt et al., 2000 \\
\hline Ferricrocina & Trichoderma virens & Zea mays & Mukherjee et al., 2018 \\
\hline Desferrioxamina & Geobacter spp. & Oryza sativa & Yuan et al., 2019 \\
\hline Coprogeno & $\begin{array}{l}\text { Penicillium chrysogenum, } \\
\text { Histoplasma capsulatum, Neu- } \\
\text { rospora crassa y Penicillium } \\
\text { nalgiovense }\end{array}$ & Cucumis sativus, N/D & $\begin{array}{l}\text { Hördt et al., 2000; Howard et al., } \\
\text { 2000; Emri et al., } 2013\end{array}$ \\
\hline $\begin{array}{l}\text { Ácido dimérico, Fusarinina } \\
\text { y Ferricrocina }\end{array}$ & $\begin{array}{l}\text { P. chrysogenum y } H \text {. capsula- } \\
\text { tum }\end{array}$ & Cucumis sativus & $\begin{array}{l}\text { Hördt et al., 2000; Howard et al., } \\
2000\end{array}$ \\
\hline Pioverdina & Pseudomonas fluorescens & Solanum lycopersicum & Nagata et al., 2013 \\
\hline Ferricromo & $\begin{array}{l}\text { Escherichia coli en Rhizobium } \\
\text { spp. }\end{array}$ & Cajanus cajan & Rajendran et al., 2007 \\
\hline Pioverdina & Pseudomonas aeruginosa & Musa spp. & Ayyadurai et al., 2006 \\
\hline Fenazina-1-carboxilato & Pseudomonas spp. & Oryza sativa & Hernández-Rodríguez et al., 2014 \\
\hline Pioverdina & Pseudomonas aeruginosa & $\begin{array}{l}\text { Cicer arietinum, Cajanus cajan y Arachis } \\
\text { hypogaea }\end{array}$ & Sulochana et al., 2014 \\
\hline Ornibactina y Pioquelina & Burkholderia spp. & $\mathrm{N} / \mathrm{D}$ & Asghar et al., 2011 \\
\hline \multicolumn{4}{|l|}{ Fitosideróforos } \\
\hline Mimosina & - & Leucaena leucocephala & Honda y Borthakur, 2020 \\
\hline Ácidos mugineicos & - & Petunia spp. & Murata et al., 2015 \\
\hline Ácido desoximugineico & - & Triticum durum & Aciksoz et al., 2011 \\
\hline
\end{tabular}

$\mathrm{N} / \mathrm{D}=$ no determinado 


\subsection{Labores culturales que mitigan los efectos tóxicos del Fe}

Las mejores producciones bananeras se logran en suelos franco-arcillosos con rendimientos de hasta 4.117 cajas/ha/ año (Azuero Gaona et al., 2020). Las labores culturales también juegan un papel importante para evitar la acumulación de Fe en la plantación, tales como actividades de limpieza de canales primarios y segundarios (Figura 1). Las buenas prácticas de manejo son las soluciones inmediatas ajustadas a la recomendación técnica de fertilización, y la motivación para el monitoreo continuo mediante análisis sistemáticos realizados a la plantación bananera. Recomendaciones que detectan y facilitan la mitigación a la presencia excesiva del Fe y otros elementos, que puedan ser absorbido por las plantas. Las actividades de campo, por ejemplo, deshije, riego, control de malezas, plagas, labranzas etc., apoyan una mayor absorción de nutrientes provenientes de la aplicación de fertilizantes al suelo (Ghosh et al., 2002; Dens et al., 2008). La preservación de la cobertura vegetal permite mayor oxigenación del suelo (Villa Guerrero et al., 2018; Chabla Carrillo et al., 2019) y la reutilización de residuos para una mayor sostenibilidad de la biodiversidad microbiana (García Batista et al., 2020), como la de los microorganismos productores de compuestos sideróforos que amortiguan los metales pesados como el Fe. El pseudotallo de banano es un recurso natural renovable que contiene Fe y puede ser utilizado en la pirólisis rápida para la producción de bioaceite (Kumar et al., 2019).
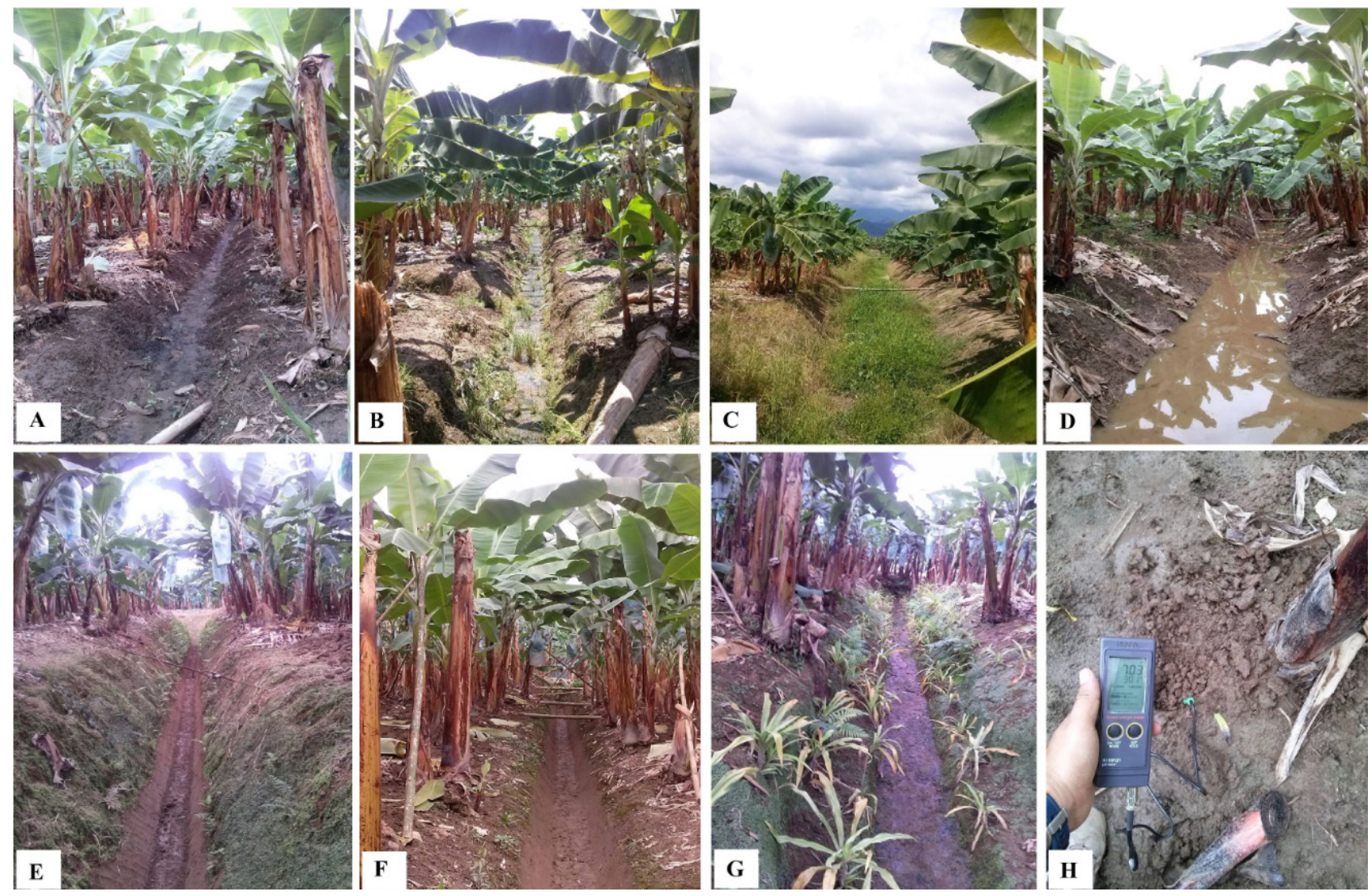

Figura 1. Problemas y medidas de mitigación en plantaciones bananeras; A-B) Acumulación de sedimentos en canales de drenaje, C) Acumulación de malezas en canales primarios, D) Estancamiento de agua en canales, E-F) Mantenimiento y limpieza de canales primarios y secundarios, G) Manejo ecológico de taludes, H) Monitoreo de $\mathrm{pH}$ en suelos.

Figure 1. Problems and mitigation measures in banana farms; $A$-B) Sediment accumulation in drainage channels, C) Weed accumulation in primary channels, D) Water stagnation in channels, E-F) Maintenance and cleaning of primary and secondary channels, G) Ecological management of banks, H) Soil pH monitoring.

La salud del agroecosistema bananero requiere de una alta tasa de evapotranspiración mensual y requiere un riego promedio de 1.418,19 mm anuales de agua; al regar se tiene que poner especial atención en suelos con mucha presencia de arcillas (Santacruz de León y Santacruz de León, 2020). Dada sus propiedades elastoplásticas los suelos arcillosos se compactan y no permiten que el agua se infiltre con facilidad, lo que produce encharcamientos a nivel superficial, del mismo modo que conduce a la proliferación de ambientes 
anaerobios; esto genera problemas en los drenajes y se traducen en disminución de la producción de banano (Eras Márquez, 2020).

La compactación del suelo es originada por factores antrópicos; el peso y el pisoteo continuo sobre la plantación, de personas o maquinarias, labores en condiciones de humedad crítica y otras actividades desarrolladas en condiciones de campo, siguen siendo una de las actividades más susceptibles a generar esa condición, en particular en los suelos de textura arcillosa (González Cueto et al., 2009). La compactación del suelo en plantaciones bananeras restringe el desarrollo de raíces, limitando a la planta en su crecimiento y anclaje, provocando volcamientos y proliferación de microorganismos patógenos, resultando crítico en las plantaciones bananeras sin cobertura vegetal (Vásquez-Castillo et al., 2019). La pérdida de $10 \mathrm{~g}$ de raíces afecta la productividad del cultivo drásticamente (Serrano, 2005); la cual también se ve afectada por la presencia de enfermedades, por ejemplo, la conocida como sigatoka negra (Mycosphaerella fijiensis Morelet) (Aguirre Forero et al. 2012), el raquitismo por nematodos fitoparásitos, entre otros problemas fitosanitarios.

\section{Conclusiones}

El Fe es un micronutriente esencial para la plantación de banano, no obstante, puede ser un enemigo silencioso, dado que en altas concentraciones puede generar problemas como la retención de $\mathrm{P}$ por lo que puede ocasionar un desequilibrio nutricional en la planta. Además, las labores culturales y las aplicaciones efectivas de fertilizantes pueden proporcionar un manejo más adecuado de la plantación, dado que mejoran la productividad del agroecosistema bananero, aun con niveles altos de Fe disponible, pues este elemento vuelve a infiltrarse a las aguas subterráneas y así favorecen en gran medida a las comunidades microbianas que dependen del Fe en los diferentes horizontes del suelo para la producción de sideróforos y fitosideróforos.

\section{Agradecimientos}

Los autores desean extender un agradecimiento al Mg. Alfredo Pihuave Nacif por su lectura crítica del manuscrito y sugerencias. Además, nos gustaría agradecer a los técnicos de campo de la finca San Valentín, señores Segundo B. Naranjo Moncada (†) y Valentín Martínez por asistirnos en las visitas a la plantación bananera.

\section{Financiamiento}

Este trabajo fue financiado por los agricultores, quienes en el rol de patrocinadores no tuvieron ningún papel en el diseño de esta investigación, recopilación, análisis de datos, decisión de publicar o elaboración de manuscrito.

\section{Contribuciones de los autores}

- Jaime Naranjo-Morán: Conceptualización, Investigación, Recursos, Visualización, Redacción-revisión y edición.

- Marcos Vera-Morales: Curación de datos, Metodología, Análisis formal, Redacción - borrador original.

- Andy Mora-González: Adquisición de fondos, Administración del proyecto, Supervisión y Validación.

\section{Referencias}

Abadía, J., Vázquez, S., Rellán-Álvarez, R., El-Jendoubi, H., Abadía, A., Álvarez-Fernández, A., \& López-Millán, A. F. (2011). Towards a knowledge-based correction of iron chlorosis. Plant Physiology and Biochemistry, 49(5), 471-482. https://doi.org/10.1016/j.plaphy.2011.01.026

Aciksoz, S. B., Ozturk, L., Gokmen, O. O., Römheld, V., \& Cakmak, I. (2011). Effect of nitrogen on root release of phytosiderophores and root uptake of Fe (III)-phytosiderophore in Fe-deficient wheat plants. Physiologia Plantarum, 142(3), 287-296. https://doi.org/10.1111/j.1399-3054.2011.01460.x 
Agrocalidad. (2015). Instructivo INT/SFA/ll. Muestreo para análisis de foliares. Rev. 2. Agrocalidad.

Aguado-Santacruz, G. A., Moreno-Gómez, B., Jiménez-Francisco, B., García-Moya, E., \& Preciado-Ortiz, R. E. (2012). Impacto de los sideróforos microbianos y fitosidéforos en la asimilación de hierro por las plantas: una síntesis. Revista Fitotecnia Mexicana, 35(1), 9-21.

Aguirre Forero, S. E., Piraneque Gambasica, N. V., \& Menjivar Flores, J. C. (2012). Relación entre las propiedades edafoclimáticas y la incidencia de sigatoka negra (Mycosphaerella fijiensis Morelet) en la zona bananera del Magdalena-Colombia. Revista de Investigación Agraria y Ambiental, 3(2), 13-25. https:// doi.org/10.22490/21456453.970

Ailloud, F., Lowe, T. M., Robène, I., Cruveiller, S., Allen, C., \& Prior, P. (2016). In planta comparative transcriptomics of host-adapted strains of Ralstonia solanacearum. PeerJ, 4, e1549. https://doi.org/10.7717/peerj.1549

Årstøl, E., \& Hohmann-Marriott, M. F. (2019). Cyanobacterial Siderophores-Physiology, Structure, Biosynthesis, and Applications. Marine Drugs, 17(5), 281. https://doi.org/10.3390/md17050281

Asghar, A. H., Shastri, S., Dave, E., Wowk, I., Agnoli, K., Cook, A. M., \& Thomas, M. S. (2011). The pobA gene of Burkholderia cenocepacia encodes a group I Sfp-type phosphopantetheinyltransferase required for biosynthesis of the siderophores ornibactin and pyochelin. Microbiology, 157(2), 349-361. https://doi. org/10.1099/mic.0.045559-0

Aulakh, M. S., \& Malhi, S. S. (2005). Interactions of nitrogen with other nutrients and water: effect on crop yield and quality, nutrient use efficiency, carbon sequestration, and environmental pollution. Advances in Agronomy, 86, 341-409. https://doi.org/10.1016/S0065-2113(05)86007-9

Ayyadurai, N., Ravindra Naik, P., Sreehari Rao, M., Sunish Kumar, R., Samrat, S. K., Manohar, M., \& Sakthivel, N. (2006). Isolation and characterization of a novel banana rhizosphere bacterium as fungal antagonist and microbial adjuvant in micropropagation of banana. Journal of Applied Microbiology, 100(5), 926-937. https://doi.org/10.1111/j.1365-2672.2006.02863.x

Azuero Gaona, B. R., Quevedo Guerrero, J. N., \& García Batista, R. M. (2020). Efecto del biocarbón y microorganismos en la producción y estado fitosanitario de banano orgánico en la parroquia "La Victoria". Revista Cientifica Agroecosistemas, 8(2), 110-120. https://aes.ucf.edu.cu/index.php/aes/article/view/408

Baque Mite, R., Simba Ochoa, L., González Osorio, B., Suatunce, P., Diaz Ocampo, E., \& Cadme Arevalo, L. (2016). Calidad del agua destinada al consumo humano en un cantón de Ecuador. CIENCIA UNEMI, 9(20), 109-117. https://doi.org/10.29076/issn.2528-7737vo19iss20.2016pp109-117p

Bustamante León, M. B., Chabla-Carrillo, C. J., \& Barrezueta-Unda, C. S. (2018). La densidad y humedad crítica como indicadores de la compactación de suelos cultivados con banano. Revista Científica Agroecosistemas, 6(1), 168-174. https://aes.ucf.edu.cu/index.php/aes/article/view/179

Caicedo Camposano, O., Balmaseda Espinosa, C., \& Proaño Saraguro, J. (2015). Programación del riego del banano (Musa paradisiaca) en finca San José 2, Los Ríos, Ecuador. Revista Ciencias Técnicas Agropecuarias, 24(2), 18-22. https://revistas.unah.edu.cu/index.php/rcta/article/view/339

Chabla Carrillo, J., Vidal Vazquez, E., Barrezueta Unda, S., \& Bustamante León, M. (2020). Determinación del intervalo Hídrico Optimo en un suelo Inceptisol bananero, bajo sistemas de riego. Revista Cientifica Agroecosistemas, 7(3), 38-44. https://aes.ucf.edu.cu/index.php/aes/article/view/313

Connorton, J. M., Balk, J., \& Rodríguez-Celma, J. (2017). Iron homeostasis in plants-a brief overview. Metallomics, 9(7), 813-823. https://doi.org/10.1039/C7MT00136C

de Deus, J. A. L., Neves, J. C. L., Corrêa, M. C. d. M., Parent, S. É., Natale, W., \& Parent, L. E. (2018). Balance design for robust foliar nutrient diagnosis of "Prata" banana (Musa spp.). Scientific Reports, 8(1), 1-7. https://doi.org/10.1038/s41598-018-32328-y

De Serrano, L. O. (2017). Biotechnology of siderophores in high-impact scientific fields. Biomolecular concepts, 8(3-4), 169-178. https://doi.org/10.1515/bmc-2017-0016

De Serrano, L. O., Camper, A. K., \& Richards, A. M. (2016). An overview of siderophores for iron acquisition in microorganisms living in the extreme. Biometals, 29(4), 551-571. https://doi.org/10.1007/s10534-0169949-x

Dens, K. R., Romero, R. A., Swennen, R., \& Turner, D. W. (2008). Removal of bunch, leaves, or pseudostem alone, or in combination, influences growth and bunch weight of ratoon crops in two banana cultivars. The Journal of Horticultural Science and Biotechnology, 83(1), 113-119. https://doi.org/10.1080/1 4620316.2008.11512355

Dong, X., Wang, M., Ling, N., Shen, Q., \& Guo, S. (2016). Effects of iron and boron combinations on the suppression of Fusarium wilt in banana. Scientific Reports, 6, 38944. https://doi.org/10.1038/srep38944 
El-Amier, Y. A., Alghanem, S. M., \& Alzuaibr, F. M. (2017). Bioaccumulation and translocation of heavy metals from coastal soil by wild halophytes. American Journal of Environmental Protection, 5(2), 52-60. https://doi.org/10.12691/env-5-2-4

Emri, T., Tóth, V., Nagy, C. T., Nagy, G., Pócsi, I., Gyémánt, G., Antal K., Balla, J., Balla, G., Román, G., Kovács, I., Pócsi, I. (2013). Towards high-siderophore-content foods: optimisation of coprogen production in submerged cultures of Penicillium nalgiovense. Journal of the Science of Food and Agriculture, 93(9), 2221-2228. https://doi.org/10.1002/jsfa.6029

Eras Márquez, R. Y. (2020). Estimación de las relaciones espaciales entre la conductividad hidráulica y las propiedades fisicas del suelo en el cultivo de banano. Universidad Técnica de Machala.

Espinosa, J., \& Mite, F. (2008). Búsqueda de eficiencia en el uso de nutrientes en banano. International Plant Nutrition Institute. XVIII Congreso ACORBAT. Guayaquil, Ecuador.

Forster, M. P., Rodríguez Rodríguez, E., Martín, J. D., \& Díaz Romero, C. (2002). Statistical differentiation of bananas according to their mineral composition. Journal of Agricultural and Food Chemistry, 50(21), 6130-6135. https://doi.org/10.1021/jf0255578

García Batista, R. M., Quevedo Guerrero, J. N., \& Socorro Castro, A. R. (2020). Prácticas para el aprovechamiento de residuos sólidos en plantaciones bananeras y resultados de su implementación. Universidad y Sociedad, 12(1), 280-291. https://rus.ucf.edu.cu/index.php/rus/article/view/1454

Gbongue, L. -R., Lalaymia, I., Zeze, A., Delvaux, B., \& Declerck, S. (2019). Increased silicon acquisition in bananas colonized by Rhizophagus irregularis MUCL 41833 reduces the incidence of Pseudocercospora fijiensis. Frontiers in Plant Science, 9, 1977. https://doi.org/10.3389/fpls.2018.01977

Ghosh, P. K., Wanjari, R. H., Mandal, K. G., Hati, K. M., \& Bandyopadhyay, K. K. (2002). Recent trends in inter-relationship of nutrients with various agronomic practices of field crops in India. Journal of Sustainable Agriculture, 21(1), 47-77. https://doi.org/10.1300/J064v21n01_06

González Cueto, O., Iglesias Coronel, C. E., \& Herrera Suárez, M. (2009). Análisis de los factores que provocan compactación del suelo agrícola. Revista Ciencias Técnicas Agropecuarias, 18(2),57-63.

Grillet, L., \& Schmidt, W. (2017). The multiple facets of root iron reduction. Journal of Experimental Botany, 68(18), 5021-5027. https://doi.org/10.1093/jxb/erx320

Hedrich, S., Schlömann, M., \& Johnson, D. B. (2011). The iron-oxidizing proteobacteria. Microbiology, 157(6), 1551-1564. https://doi.org/10.1099/mic.0.045344-0

Herlihy, J. H., Long, T. A., \& McDowell, J. M. (2020). Iron homeostasis and plant immune responses: Recent insights and translational implications. Journal of Biological Chemistry, 295(39), 13444-13457. https:// doi.org/10.1074/jbc.REV120.010856

Hernández-Rodríguez, A., Rives-Rodríguez, N., Acebo-Guerrero, Y., Díaz-de la Osa, A., Heydrich-Pérez, M., \& Divan Baldani, V. L. (2014). Potencialidades de las bacterias diazotróficas asociativas en la promoción del crecimiento vegetal y el control de Pyricularia oryzae (Sacc.) en el cultivo del arroz (Oryza sativa L.). Revista de Protección Vegetal, 29(1), 1-10.

Honda, M. D. H., \& Borthakur, D. (2020). Mimosine facilitates metallic cation uptake by plants through formation of mimosine-cation complexes. Plant Molecular Biology, 102(4), 431-445. https://doi.org/10.1007/ s11103-019-00956-1

Hördt, W., Römheld, V., \& Winkelmann, G. (2000). Fusarinines and dimerum acid, mono-and dihydroxamate siderophores from Penicillium chrysogenum, improve iron utilization by strategy I and strategy II plants. Biometals, 13, 37-46. https://doi.org/10.1023/A:1009234612486

Howard, D. H., Rafie, R., Tiwari, A., \& Faull, K. F. (2000). Hydroxamate siderophores of Histoplasma capsulatum. Infection and Immunity, 68(4), 2338-2343. https://doi.org/10.1128/IAI.68.4.2338-2343.2000

Huang, C., Liu, S., Li, R., Sun, F., Zhou, Y., \& Yu, G. (2016). Spectroscopic evidence of the improvement of reactive iron mineral content in red soil by long-term application of swine manure. PloS One, 11(1), e0146364. https://doi.org/10.1371/journal.pone.0146364

Instituto Nacional de Investigaciones Agropecuarias [INIAP], (2013). Servicio de diagnóstico de problemas fitosanitarios para el sector agrícola del país. Plegable №. 271. INIAP, Estación Experimental Litoral Sur "Dr. Enrique Ampuero Pareja", Departamento Nacional Protección Vegetal.

Izquierdo, M., \& Armas, M. (2018). Propuesta de un protocolo de fertilización como una estrategia para el control de nematodos en el cultivo de banano. Revista Cientifica Ciencias Naturales y Ambientales, 12(1), 31-42.

Karimian, N., Johnston, S. G., \& Burton, E. D. (2018). Iron and sulfur cycling in acid sulfate soil wetlands under dynamic redox conditions: A review. Chemosphere, 197, 803-816. https://doi.org/10.1016/j.chemosphere.2018.01.096 
Kesaulya, H., Hasinu, J. V., \& Tuhumury, G. NC. (2018). Potential of Bacillus spp produces siderophores insuppressing thewilt disease of banana plants. IOP Conference Series: Earth and Environmental Science, 102, 012016. https://doi.org/10.1088/1755-1315/102/1/012016

Khatua, C., Sengupta, S., Balla, V. K., Kundu, B., Chakraborti, A., \& Tripathi, S. (2018). Dynamics of organic matter decomposition during vermicomposting of banana stem waste using Eisenia fetida. Waste Management, 79, 287-295. https://doi.org/10.1016/j.wasman.2018.07.043

Kramer, J., Özkaya, Ö., \& Kümmerli, R. (2019). Bacterial siderophores in community and host interactions. Nature Reviews Microbiology, 18, 152-163. https://doi.org/10.1038/s41579-019-0284-4

Kumar, A., Mylapilli, S. P., \& Reddy, S. N. (2019). Thermogravimetric and kinetic studies of metal (Ru/Fe) impregnated banana pseudo-stem (Musa acuminate). Bioresource Technology, 285, 121318. https://doi. org/10.1016/j.biortech.2019.121318

Kumar, G. B. S., Srinivas, L., Ganapathi, T. R. (2011). Iron fortification of banana by the expression of soybean ferritin. Biological Trace Element Research, 142(2), 232-241. https://doi.org/10.1007/s12011-010-8754-6

Lahav, E., \& Turner, D. W. (1992). Fertilización de banano para rendimientos altos (2 ${ }^{\mathrm{a}}$ ed.). Boletín $\mathrm{N}^{\mathrm{o}} 7$, Instituto de la potasa y el fósforo.

López, A., \& Espinoza, J. (1995). Manual de nutrición y fertilización del banano. Una visión práctica del manejo de la fertilización. International Plant Nutrition Institute.

Lora Silva, R. (2007). Contaminación por elementos menores y posibles soluciones. Revista U.D.C.A Actualidad \& Divulgación Científica, 10(1), 5-20. https://doi.org/10.31910/rudca.v10.n1.2007.559

Madaan, G., Gosal, S. K., Gosal, S. S., Saroa, G. S., \& Gill, M. I. S. (2013). Effect of microbial inoculants on the growth and yield of micropropagated banana (Musa indica) cv. Grand Naine. The Journal of Horticultural Science and Biotechnology, 88(5), 643-649. https://doi.org/10.1080/14620316.2013.11513019

Maldonado-Magaña, A., Favela-Torres, E., Rivera-Cabrera, F., \& Volke-Sepulveda, T. L. (2011). Lead bioaccumulation in Acacia farnesiana and its effect on lipid peroxidation and glutathione production. Plant and Soil, 339(1), 377-389. https://doi.org/10.1007/s11104-010-0589-6

Martínez, R. E., Smith, D. S., Pedersen, K., \& Ferris, F. G. (2003). Surface chemical heterogeneity of bacteriogenic iron oxides from a subterranean environment. Environmental Science \& Technology, 37(24), 5671-5677. https://doi.org/10.1021/es0342603

Medina Domínguez, E. K. (2006). Estudios de suelos, nutrición y fertilización en varias zonas bananeras del Ecuador. X Congreso Ecuatoriano de la Ciencia del Suelo, Guayaquil, Ecuador.

Mukherjee, P. K., Hurley, J. F., Taylor, J. T., Puckhaber, L., Lehner, S., Druzhinina, I., Schumacher, R., \& Kenerley, C. M. (2018). Ferricrocin, the intracellular siderophore of Trichoderma virens, is involved in growth, conidiation, gliotoxin biosynthesis and induction of systemic resistance in maize. Biochemical and Biophysical Research Communications, 505(2), 606-611. https://doi.org/10.1016/j.bbrc.2018.09.170

Murata, Y., Itoh, Y., Iwashita, T., \& Namba, K. (2015). Transgenic petunia with the iron (III)-phytosiderophore transporter gene acquires tolerance to iron deficiency in alkaline environments. PLoS One, 10(3), e0120227. https://doi.org/10.1371/journal.pone.0120227

Nagata, T., Oobo, T., \& Aozasa, O. (2013). Efficacy of a bacterial siderophore, pyoverdine, to supply iron to Solanum lycopersicum plants. Journal of Bioscience and Bioengineering, 115(6), 686-690. https://doi. org/10.1016/j.jbiosc.2012.12.018

Olivares, B. O., Gómez, J. A., Landa, B. B., Rey, J. C., Lobo, D., \& Navas, J. A. (2018) Análisis de la calidad de suelos tropicales sobre la productividad del banano en diferentes fincas de los estados de Aragua y Trujillo en Venezuela. Congreso Nacional de Medio Ambiente - CONAMA 2018. Madrid, España.

Oliveira, S. R., Menegário, A. A., \& Arruda, M. A. Z. (2014). Evaluation of Fe uptake and translocation in transgenic and non-transgenic soybean plants using enriched stable ${ }^{57} \mathrm{Fe}$ as a tracer. Metallomics, $6(10)$, 1832-1840. https://doi.org/10.1039/C4MT00162A

Pardha-Saradhi, P., Yamal, G., Peddisetty, T., Sharmila, P., Singh, J., Nagarajan, R., \& Rao, K. S. (2014). Plants fabricate Fe-nanocomplexes at root surface to counter and phytostabilize excess ionic Fe. Biometals, 27(1), 97-114. https://doi.org/10.1007/s10534-013-9690-7

Pérez-López, E. (2013). Análisis de fertilidad de suelos en el laboratorio de Química del Recinto de Grecia, Sede de Occidente, Universidad de Costa Rica. InterSedes, 14(29), 06-18. https://doi.org/10.15517/isucr.v14i29.13496

Phirke, N. V., Kothari, R. M., \& Chincholkar, S. B. (2008). Rhizobacteria in mycorrhizosphere improved plant health and yield of banana by offering proper nourishment and protection against diseases. Applied Biochemistry and Biotechnology, 151(2-3), 441. https://doi.org/10.1007/s12010-008-8212-5 
Pi, H., \& Helmann, J. D. (2017). Ferrous iron efflux systems in bacteria. Metallomics, 9(7), 840-851. https:// doi.org/10.1039/C7MT00112F

Raghupathi, H. B., Reddy, B. M. C., \& Srinivas, K. (2002). Multivariate diagnosis of nutrient imbalance in banana. Communications in Soil Science and Plant Analysis, 33(13-14), 2131-2143. https://doi.org/10.1081/ CSS-120005753

Rajendran, G., Mistry, S., Desai, A. J., \& Archana, G. (2007). Functional expression of Escherichia coli fhuA gene in Rhizobium spp. of Cajanus cajan provides growth advantage in presence of $\mathrm{Fe}^{3+}$ : ferrichrome as iron source. Archives of Microbiology, 187(4), 257. https://doi.org/10.1007/s00203-006-0191-8

Rietra, R. P. J. J., Heinen, M., Dimkpa, C. O., \& Bindraban, P. S. (2017). Effects of nutrient antagonism and synergism on yield and fertilizer use efficiency. Communications in Soil Science and Plant Analysis, 48(16), 1895-1920. https://doi.org/10.1080/00103624.2017.1407429

Rodríguez, M., Rey, R., \& Sarmiento, O. (2005). Influencia del riego por goteo superficial y subterráneo sobre la distribución radical del banano. Revista Ciencias Técnicas Agropecuarias, 14(2), 44-48.

Sahodaran, N. K., \& Ray, J. G. (2018). Heavy metal contamination in "chemicalized" green revolution banana fields in southern India. Environmental Science and Pollution Research, 25(27), 26874-26886. https:// doi.org/10.1007/s11356-018-2729-0

Sancho, H., \& Molina, E. (2016). Efecto de la concentración del H3O+ y Mg en el crecimiento inicial y la absorción de nutrimentos en plantas de banano cultivadas en solución hidropónica. Siembra, 3(1), 37-52. https://doi.org/10.29166/siembra.v3i1.257

Santacruz de León, G., \& Santacruz de León, E. E. (2020). Evaluación del desempeño del riego por aspersión en lotes con cultivo de banana en Chiapas, México. Siembra, 7(2), 001-013. https://doi.org/10.29166/ siembra.v7i2.1712

Santos, M. S., Nogueira, M. A., \& Hungria, M. (2019). Microbial inoculants: reviewing the past, discussing the present and previewing an outstanding future for the use of beneficial bacteria in agriculture. $A M B$ Express, 9, 205. https://doi.org/10.1186/s13568-019-0932-0

Serrano, E. (2005). Relationship between functional root content and banana yield in Costa Rica. In D. W. Turner y F. E. Rosales (eds.) Banana root system: towards a better understanding for its productive management. Proceedings of an International Symposium held in San José, Costa Rica on 3-5 November 2003 (pp. 25-34). International Network for the Improvement of Banana and Plantain [INIBAP].

Sulochana, M. B., Jayachandra, S. Y., Kumar, S. A., Parameshwar, A. B., Reddy, K. M., \& Dayanand, A. (2014). Siderophore as a potential plant growth-promoting agent produced by Pseudomonas aeruginosa JAS-25. Applied Biochemistry and Biotechnology, 174(1), 297-308. https://doi.org/10.1007/s12010-0141039-3

Sulu-Gambari, F., Seitaj, D., Meysman, F. J., Schauer, R., Polerecky, L., \& Slomp, C. P. (2016). Cable bacteria control iron-phosphorus dynamics in sediments of a coastal hypoxic basin. Environmental Science \& Technology, 50(3), 1227-1233. https://doi.org/10.1021/acs.est.5b04369

Tan, W., Yuan, Y., Zhao, X., Dang, Q., Yuan, Y., Li, R., Cui, D., \& Xi, B. (2019). Soil solid-phase organic matter-mediated microbial reduction of iron minerals increases with land use change sequence from fallow to paddy fields. Science of the Total Environment, 676, 378-386. https://doi.org/10.1016/j.scitotenv.2019.04.288

Tsai, H. H., \& Schmidt, W. (2020). pH-dependent transcriptional profile changes in iron-deficient Arabidopsis roots. BMC genomics, 21, 694. https://doi.org/10.1186/s12864-020-07116-6

Usuga Osorio, C. E., Castañeda Sánchez, D. A., Franco Molano, A. E., Gómez Velásquez, F. A., \& Lopera Agudelo, C. A. (2008). Efecto de la micorrización y la fertilización en la acumulación de biomasa en plantas de banano (Musa AAA cv. Gran Enano) (Musaceae). Revista Facultad Nacional de Agronomía Medellín, 61(1), 4269-4278. https://revistas.unal.edu.co/index.php/refame/article/view/24726

Van Groeningen, N., ThomasArrigo, L. K., Byrne, J. M., Kappler, A., Christl, I., \& Kretzschmar, R. (2020). Interactions of ferrous iron with clay mineral surfaces during sorption and subsequent oxidation. Environmental Science: Processes \& Impacts 22, 1355-1367. https://doi.org/10.1039/D0EM00063A

Vásquez-Castillo, W., Racines-Oliva, M., Moncayo, P., Viera, W., \& Seraquive, M. (2019). Calidad del fruto y pérdidas poscosecha de banano orgánico Musa acuminata en el Ecuador. Enfoque UTE, 10(4), 57-66. https://doi.org/10.29019/enfoque.v10n4.545

Villa Guerrero, I. P. E., Chabla Carrillo, D. C. J. E., \& Villaseñor Ortiz, M. D. R. (2018). Efecto de riegos presurizados sobre propiedades físicas de un suelo bananero asociado con Kudzu (Pueraria phaseoloi- 
des Benth). Revista Cientifica Agroecosistemas, 6(1), 34-45. https://aes.ucf.edu.cu/index.php/aes/article/ view/162

Voß, B., Kirschhöfer, F., Brenner-Weiß, G., \& Fischer, R. (2020). Alternaria alternata uses two siderophore systems for iron acquisition. Scientific Reports, 10(1), 3587. https://doi.org/10.1038/s41598-020-60468-7

Wang, Z., Li, R., Cui, L., Fu, H., Lin, J., \& Chen, J. (2018). Characterization and acid-mobilization study for typical iron-bearing clay mineral. Journal of Environmental Sciences, 71, 222-232. https://doi.or$\mathrm{g} / 10.1016 / \mathrm{j} . j \mathrm{es} .2018 .04 .012$

Yuan, C., Li, F., Cao, W., Yang, Z., Hu, M., \& Sun, W. (2019). Cadmium solubility in paddy soil amended with organic matter, sulfate, and iron oxide in alternative watering conditions. Journal of Hazardous Materials, 378, 120672. https://doi.org/10.1016/j.jhazmat.2019.05.065

Zielińska-Dawidziak, M. (2015). Plant ferritin-A source of iron to prevent its deficiency. Nutrients, 7(2), 1184-1201. https://doi.org/10.3390/nu7021184 